\title{
INTERVIEW
}

\section{With Jens H. Petersen, author of The Kingdom of Fungi ${ }^{1}$}

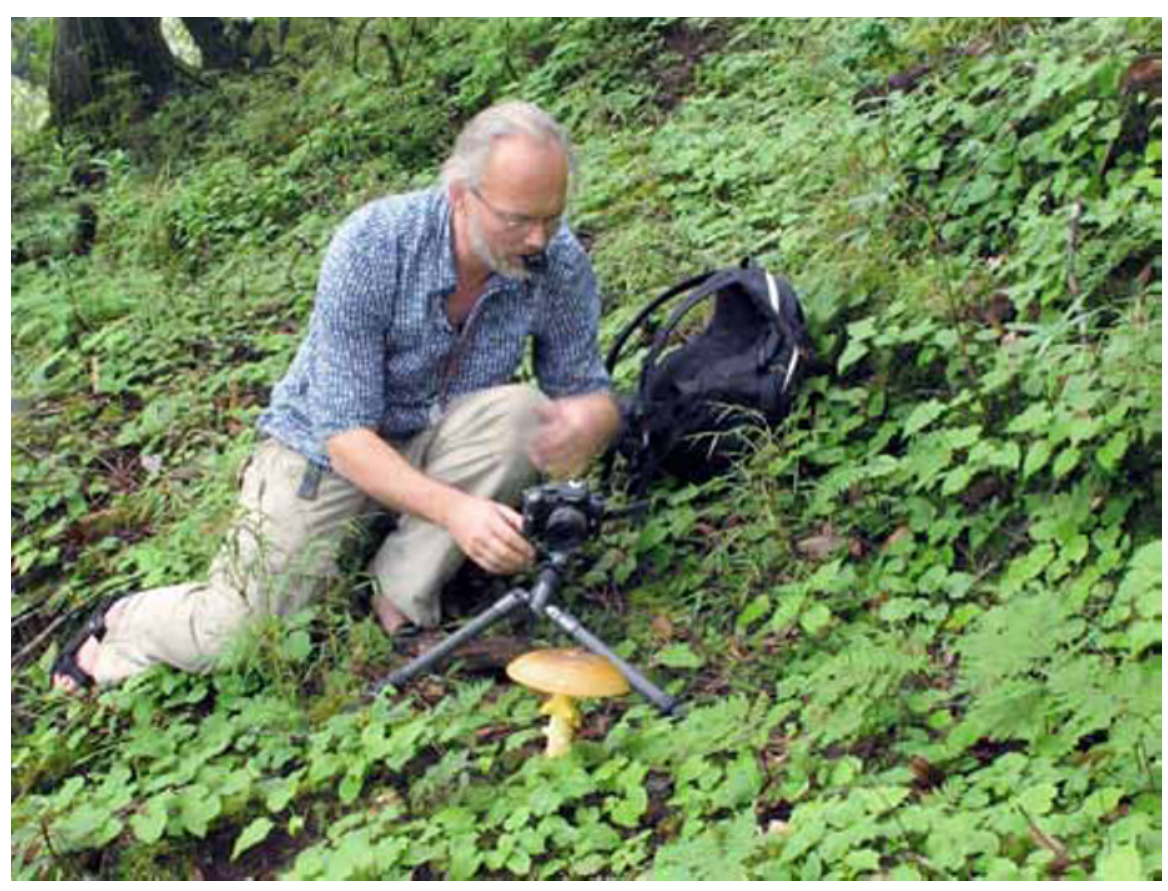

The fungal community has recently been buzzing about the new book recently published by Jens Petersen, which fills most mycologists with awe, and again underlines the wonders of the magical kingdom of Fungi. However, many of us have been asking ourselves, who is Jens Petersen, and how exactly did he capture these incredible photographs? To help answer some of these questions, we managed to track him down in Spain, where he just returned from a fungal ascomycete (MycoAsco) workshop. Sitting at the airport waiting for his flight back to Denmark was perfect, as Jens had time to give us some insight into his world.

\section{Who is Jens H. Petersen?}

I am a mycologist and photographer living near Aarhus, Denmark. For 20 years I taught mycology at the University of Aarhus, but now I am a freelance mycologist and author/photographer. I was originally educated as a fungal taxonomist but found a broader perspective more interesting than solving species delimitation in Ramaria. Together with my good friend and college Thomas Læssøe, back in 1998 we started

${ }^{1}$ See Book News in this issue of IMA Fungus, p. (26). the genus identification project MycoKey (www.mycokey.com). This is exactly the kind of project that allows me to dig around in all possible corners of mycology - and a target for every good fungus photograph I can produce.

\section{Why did you write this book?}

When I was teaching at the university I very much wanted a better textbook than those available. Thus, in 1995, I produced my own book Svamperiget (transl. The Fungal Kingdom) illustrated with lots of black and white photographs and line drawings. As the book was in Danish, it was much appreciated by the Danish students, but they always complained about the lack of colour. My friends in the local mycological society, on the other hand, claimed it was an excellent book to keep beside the bed - it made them fall asleep very fast! So, for some years I played with the idea of compiling a colourful and much more seductive book dealing with fungi and their lifestyles.

I think most professional mycologists have experienced how hard it is to get Fungi recognised as equal to Plants and Animals. This is partly due to tradition, and partly due to the lack of a broader recognition of
Fungi as a unique kingdom. The bookstores are filled with books on edible and poisonous fungi (I have written a couple myself), but there was a great need for a book that could visualize the unique life of fungi. We have to insist that fungi are not "Lower Plants", their occurrence in nature should not be called flora but funga, they are not kept in herbaria but in fungaria, etc. We have to insist on their uniqueness, their importance, their great number and the need to explore them - the book is an attempt to do this...

\section{Why so many pictures, and what is the philosophy behind it?}

As this book is mainly aimed towards the nature-interested non-mycologist, I wanted the book to have the smallest possible amount of text, and - through the illustrations - to be as self explanatory as possible. I think that the first introduction to the fungi - whether for students or the broader public - works best in this more intuitive way.

\section{How did you select the different taxa to include? Is there a story behind it, or did} you simply take what you came across? I wanted to show fungal diversity by presenting as many of the fungal form groups as possible. That part was easy, as I had already made loads of pictures in connection with MycoKey and on various expeditions to for example Greenland, Ecuador, Burkina Faso, and Bhutan. But I also wanted to present fungal biology and ecology and this was much harder to do through photographs. I worked for a couple of years photographing mycelia, hyphae, asexual morphs, etc. to prepare this part of the book.

\section{Do you ever culture these fungi?}

No, I haven't really got the facilities and patience for this.

\section{Who did the layout of the book, and the graphics?}

I did the layout and graphics myself. I have been doing book and journal layout since desktop publishing arose in the nineties 
and I obtain great satisfaction working directly in layout mode, so that I can get the illustrations and text to interact in the optimal way.

Which cameras do you use in the field? Are there specific settings, and lenses? Any other materials? It looks like you use a flash a lot. The pictures in the book are made with a range of cameras from an ancient Nikkormat from the seventies to modern digital cameras. I especially love to use small digital cameras with articulated screens (like the Coolpix 995 and 7700). The way these cameras allow you to work very low, even underneath the sporing bodies which gives a lot of fresh angles to the illustrations and the cameras are very fast to work with. The smaller pixel counts of so called "prosumer" cameras doesn't bother me, since pictures from even the old 3.5 MP Nikon CoolPix 995 can be printed in A4 (the background Deflexula of the contents page of the book is exactly such a picture made in 2004 in Ecuador on a day when the more modern cameras had given up due to condensation). I very rarely use a flash in the field, but a piece of white paper to reflect light onto the darker areas is one of my best "tools". This, combined with a small aperture, a tripod and the built-in self-release, will get you far.

How did you take photos in the laboratory? And again, which cameras did you use? For lab work, MycoKey owns a Leica Macroscope purchased in 1998 which we continuously optimized with better cameras, led light, etc. At the moment I shoot with flashes through a swan neck and use a Nikon d 90 controlled from a Mac on top of the scope.

Do you enhance these photos via Photoshop, or are they simply that good when you first take them?

All photographs are optimized in

Photoshop. Even the best cameras rarely get the colours just right, and in the end, pictures must be scaled, colour separated and sharpened. One of the latest software trends is stacking: you take a great number of pictures with different focus and the software integrates it all into one picture with a very large depth of field. This is especially useful when photographing very small fungi (like inoperculate discomycetes) and I use it a lot.

Can we expect a sequel to this book, or was this the one and only?

This book is a finished project, but there may be others ahead. We (Thomas Læssøe and I) would very much like to do a coffee table book on amazonian fungi so the public could be seduced by tropical fungi the way they are by coloured frogs, birds and insects. We still need an opportunity to make more pictures, but there is a small teaser from Ecuador (alas in Danish) available as a pdf on the MycoKey webpage.

Pedro Crous

(p.crous@cbs.knaw.nl)

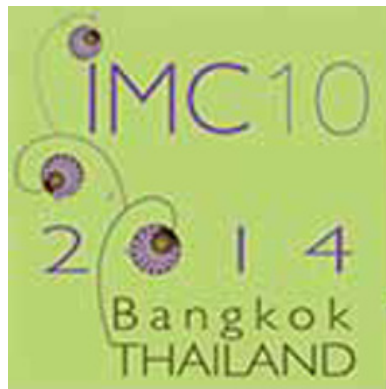

The $10^{\text {th }}$ International Mycological Congress in Bangkok, THAILAND AUGUST 3 TO 8, 2014

www.imc10.kasetsart.org 